\title{
Schistosoma japonicum cathepsin B as potential diagnostic antigen for Asian zoonotic schistosomiasis
}

\author{
Adrian Miki C. Macalanda ${ }^{1,2} \cdot$ Jose Ma. M. Angeles ${ }^{1,3} \cdot$ Kharleezelle J. Moendeg $^{1,4} \cdot$ Minh-Anh Dang-Trinh ${ }^{1,5}$. \\ Luna Higuchi $^{1}$ • Masashi Kirinoki ${ }^{6}$. Yuichi Chigusa ${ }^{6}$ - Lydia R. Leonardo ${ }^{3}$ • Elena A. Villacorte ${ }^{3}$. Pilarita T. Rivera ${ }^{3}$. \\ Yasuyuki Goto ${ }^{7}$. Shin-ichiro Kawazu ${ }^{1}$
}

Received: 28 February 2019 / Accepted: 25 July 2019/Published online: 3 August 2019

(C) Springer-Verlag GmbH Germany, part of Springer Nature 2019

\begin{abstract}
In this study, the diagnostic value of Schistosoma japonicum cathepsin B (SjCatB) was evaluated as an antigen for the early detection of $S$. japonicum infection. SjCatB is a key protease used by the cercaria to penetrate the intact skin of the host for transdermal infection. The early exposure of the host's immune system to this enzyme may elicit early production of antibodies against this molecule. Therefore, the recombinant SjCatB (rSjCatB) was expressed in Escherichia coli with N-terminal 6xHistag. $\mathrm{rSjCatB}$ was tested for its performance as a diagnostic antigen using indirect enzyme-linked immunosorbent assay (ELISA) with sera from experimentally infected mice collected at $>8$ weeks post-infection. Showing $100 \%$ sensitivity and $95.0 \%$ specificity in the ELISA, rSjCatB was then evaluated with sera from experimentally infected mice collected at 1-7 weeks post-infection to determine how early the antibodies can be detected. Results showed that as early as 6 weeks post-infection, 2 of the 3 infected mice were found to be positive with the antibodies against SjCatB. Furthermore, the potential of the recombinant antigen in detecting human schistosomiasis was evaluated with archived serum samples collected from individuals who had been diagnosed with $S$. japonicum infection by stool examination. Results showed $86.7 \%$ sensitivity and $96.7 \%$ specificity suggesting its high diagnostic potential for human schistosomiasis. In addition, SjCatB showed minimal cross-reaction with the sera collected from patients with other parasitic diseases. In conclusion, the results of this study suggest that $\mathrm{SjCatB}$ will be useful in the development of a sensitive and specific early detection test for S. japonicum infection.
\end{abstract}

Keywords Cathepsin B · Diagnosis · ELISA · Schistosoma japonicum

Section Editor: Xing-Quan Zhu

Electronic supplementary material The online version of this article (https://doi.org/10.1007/s00436-019-06410-x) contains supplementary material, which is available to authorized users.

Shin-ichiro Kawazu

skawazu@obihiro.ac.jp

1 National Research Center for Protozoan Diseases, Obihiro University of Agriculture and Veterinary Medicine, Inada-cho,

Obihiro, Hokkaido 080-8555, Japan

2 Department of Immunopathology and Microbiology, College of Veterinary Medicine and Biomedical Sciences, Cavite State University, Cavite, Philippines

3 Department of Parasitology, College of Public Health, University of the Philippines-Manila, Manila, Philippines
4 Department of Biology, School of Science and Engineering, Ateneo de Manila University, Quezon City, Philippines

5 The United Graduate School of Veterinary Sciences, Gifu University, Gifu, Japan

6 Department of Tropical Medicine and Parasitology, Dokkyo Medical University, Tochigi, Japan

Graduate School of Agricultural and Life Sciences, The University of Tokyo, Tokyo, Japan 


\section{Introduction}

Schistosomiasis is one of the neglected tropical diseases (NTDs) that causes chronic debilitating illness in infected individuals affecting millions of people globally (Chitsulo et al. 2000). In tropical countries, the socioeconomic and public health impact of the disease is second only to malaria (CDC 2017). Schistosoma japonicum, the most prevalent species in Asia, is found in China, the Philippines, and some parts of Indonesia (Chitsulo et al. 2000). Due to the huge number of eggs excreted by the female worm, S. japonicum is considered to be the most pathogenic among the schistosomes that are infective to humans (Mahmoud 2001). Recent reports of emergence of new endemic foci being provable to be attributed to the migration of infected individuals or animals indicate that new cases of human schistosomiasis pose a threat to the elimination of the disease (Leonardo et al. 2015; Patz et al. 2000). The lack of effective screening method for humans and animals from endemic areas could lead to the introduction of the disease in non-endemic areas with environmental and ecological conditions that will satisfy the life cycle of the parasite.

A good diagnostic test that can be employed in the surveillance of schistosomiasis is that it is capable of detecting early cases of the disease. The early diagnosis of infection may prove useful especially in travelers potentially exposed to $S$. japonicum infection, where prompt treatment will definitely result in a positive outcome by killing the worms prior to egg deposition in vital organs and tissues (Karcher et al. 2008). Pathologic lesions associated with $S$. japonicum infection that mainly occur due to the deposition of eggs in various vital organs (Mahmoud 2001) may be avoided.

Being the first stage of the schistosome parasite to be exposed to the host's immune system, the cercaria seems to be useful in providing the candidate antigens that might have the potentials for the early diagnosis of the disease. Certain antibodies against the antigens related to the cercaria have been detected early in the course of infection (Zhou et al. 2010). Soluble cercarial antigen (SCA) was previously reported to be a good early diagnostic antigen (Zhang et al. 2015). However, due to the nature of SCA being a crude antigen, there are difficulties associated with its mass production for field application, and cross-reaction with other parasitic infections has been observed (Zhou et al. 2010). S. japonicum cathepsin B ( $\mathrm{SjCatB})$ is a protease expressed throughout all life stages of the parasite. The highest level of expression was observed in the infective cercaria stage in which it is utilized by the parasite to penetrate the intact skin of the host. This important role of $\mathrm{SjCatB}$ in the parasite has made it an ideal target for vaccine and drug development (Liu et al. 2014; Curwen and Wilson 2003; Dvorak et al. 2008). Although S. mansoni cathepsin B has been evaluated for the serological diagnosis of S. japonicum and S. mansoni infection (Ruppel et al. 1987; González et al. 2015), the use of SjCatB as an authentic antigen for $S$. japonicum may provide a more sensitive and specific diagnostic target.

In the present study, recombinant $\mathrm{SjCatB}$ (rSjCatB) was produced and evaluated as a diagnostic antigen using indirect enzyme-linked immunosorbent assay (ELISA).

\section{Materials and methods}

\section{Ethics statement}

The collection of serum samples from patients in Sorsogon, the Philippines, were carried out in compliance with the ethical clearance sought from the University of the Philippines, Manila (clearance no: UPM REB 2011-098), which includes informed consent from the individuals involved in the study. The animal experiments were performed under the guidelines for animal experimentation of Obihiro University of Agriculture and Veterinary Medicine (permission number: 29-52).

\section{Mice and rabbit serum samples}

A total of 5 female, 8-week-old ICR mice (Clea Inc., Tokyo, Japan) were used to observe the antibody response against $\mathrm{SjCatB}$ in the course of the infection. Three of the 5 mice were percutaneously infected with 30 cercariae using S. japonicum Yamanashi strain obtained from the Department of Tropical Medicine and Parasitology, Dokkyo Medical University, Japan. Serum samples were collected from the experimental infection $(n=3)$ at 1 -week interval for 7 weeks after the cercarial challenge. The animals were sedated using isoflurane, and blood was drawn from the dorsal tail vein using a G26 butterfly needle attached to a microhematocrit tube. The same sampling time was observed for the serum collection in 2 uninfected mice serving as negative controls. To determine the diagnostic value of $\mathrm{SjCatB}$ in experimental infection, a total of 40 mice were used. Twenty mice $(n=20)$ out of the 40 were infected with the parasite by percutaneous challenge with 30 cercariae, and serum samples from these mice were collected at $>8$ weeks post-infection. Negative controls used were serum samples collected from uninfected mice $(n=20)$. Rabbit serum samples used for western blotting were derived from 2 Japanese white rabbit Icl strain (Ichikawa-ya, Chiba, Japan). One of the rabbits was infected with 2000 cercariae by a percutaneous route which was used as the positive control, while the other non-infected rabbit was used as the negative control. The serum samples from both rabbits were collected 11 weeks post-infection. Additionally, a mouse infected with 30 cercariae served as positive control, while the serum from the other non-infected mouse was used as a negative control. Serum samples were collected at 8 weeks post-infection. Upon termination of the experiment, all mice were humanely 
sacrificed by cervical dislocation following isoflurane administration and the rabbits were sacrificed following the overdosage of sodium pentobarbital administered intravenously.

\section{Human serum samples}

Archived serum samples from patients $(n=30)$ who had been confirmed by stool microscopy and a stool PCR for $S$. japonicum infection (Angeles et al. 2012) were used in this experiment. These samples were collected from Sorsogon, Philippines. Likewise, archived serum samples were used as negative controls. These were collected from US volunteers $(n=30)$ who had not previously visited $S$. japonicum endemic areas. To check the cross-reactivity of the antigen with other parasitic infections, archived serum samples from humans infected with Trichuris trichiura $(n=1)$, Paragonimus westermani $(n=11)$, Opisthorchis viverrini $(n=8)$, and Entamoeba histolytica $(n=10)$ were used.

\section{Recombinant protein preparation}

The complete coding sequence of $\mathrm{SjCatB}$ was retrieved from the $S$. japonicum genome database with the accession number X70968.1. Total RNA was extracted from $50 \mathrm{mg}$ of the frozen adult worm by homogenizing it in $1 \mathrm{~m} l$ of TRIzol ${ }^{\circ}$ reagent (Life Technologies, Inc., Frederic, MD, U.S.A.). cDNA was synthesized using PrimeScript ${ }^{\mathrm{TM}}$ (Takara Bio. Inc., Shiga, Japan). The double-stranded full-length coding sequence was amplified by a PCR with the following primers: $5^{\prime}$-GC GGA TCC ATG TTG AAA ATC GCA GTT-3' (underline indicates the Bam HI site) and 5'-GC CTC GAG TTA GGT TTT TAT AAG TCC AG-3' (underline indicates the Xho I site). The PCR product was cloned into the pCR 2.1-TOPO vector (Invitrogen, Carlsbad, CA, USA). The identity of the cloned sequence with the sequence deposited in the database was confirmed by sequencing using an ABI Prism 3100 Genetic Analyzer (Applied Biosystems, Carlsbad, CA, USA). The coding sequence was then double-digested with Bam HI and Xho I and was subcloned into pET-28a (+) vector (Novagen, Madison, WI, USA). The recombinant protein with 6xHis-tag on the $\mathrm{N}$ terminal was expressed using Escherichia coli (BL21) cells (Takara Bio) grown in Luria Bertani broth (Sigma-Aldrich, St. Louis, MO, USA) with kanamycin $(50 \mu \mathrm{g} / \mathrm{m} l)$. The rSjCatB was purified with affinity chromatography using an Ni-NTA protein purification kit (Qiagen, Hilden, Germany) under denaturing conditions, and then the protein was dialyzed against cold phosphate-buffered saline (PBS) (pH 7.4) prior to use. The recombinant protein was quantified using a Pierce ${ }^{\mathrm{TM}}$ BCA protein assay kit (Thermo Fisher Scientific, Rockford, IL, USA), and its purity was confirmed by sodium dodecyl sulfate polyacrylamide gel electrophoresis (SDS-PAGE).

\section{Western blotting}

Two hundred nanograms of $\mathrm{rSjCatB}$ was resolved by $12 \%$ SDS-PAGE under non-reducing conditions. Sample buffer and the polypeptides were transferred onto a polyvinylidene difluoride (PVDF) membrane. The membrane strips were blocked with 5\% skim milk in PBS overnight at $4{ }^{\circ} \mathrm{C}$ with constant shaking. Excess blocking buffer was removed by washing twice with PBS containing $0.05 \%$ Tween 20 (PBS$\mathrm{T})$. The strips were incubated with antibody or serum samples in separate reaction chambers for $1 \mathrm{~h}$ at room temperature with constant shaking. The monoclonal antibody $(\mathrm{mAb})$ against 6xHis-tag (Thermo Fisher Scientific) was diluted 500 times using $1 \%$ bovine serum albumin with PBS-T (1\%-BSA-PBS$\mathrm{T}$ ), while the mouse and rabbit sera were diluted 400 times with PBS-T. Human serum was diluted 200 times with 1\%BSA-PBS-T. After the incubation, the strips were washed three times with PBS-T and incubated with secondary antibody for either $1 \mathrm{~h}$ against mouse $\mathrm{IgG}$, rabbit IgG, or human IgG conjugated with horseradish peroxidase (HRP) (GE Healthcare, Buckinghamshire, UK). The antigen-antibody reaction was visualized by chemiluminescence using SuperSignal $^{\mathrm{TM}}$ (Thermo Fisher Scientific) and a chemiluminescent detection machine ImageQuant LAS 500 (GE Electric, Tokyo, Japan). Similarly, in order to determine the antigenicity of $\mathrm{rSjCatB}, 200 \mathrm{ng}$ of cercarial lysate was resolved by SDS-PAGE and the polypeptides were transferred onto a PVDF membrane. The polypeptides were then probed by antibodies collected from a mouse immunized with rSjCatB.

\section{ELISA}

Each well of a 96-well polystyrene plate (Thermo Fisher Scientific) was coated with $200 \mathrm{ng}$ of $\mathrm{rSjCatB}$ in $100 \mu \mathrm{l}$ of carbonate/bicarbonate buffer $(\mathrm{pH} 9.6)$ overnight at $4{ }^{\circ} \mathrm{C}$. The wells were washed three times with PBS to remove unbound antigens. The wells were blocked with $1 \%$-BSA-PBS-T for $5 \mathrm{~min}$. The mouse sera were diluted 200 times, while the human sera were diluted 400 times in 1\%-BSA-PBS-T. For each sample, $100 \mu \mathrm{l}$ of the diluted serum sample was delivered to each well in triplicate. The wells were incubated for $1 \mathrm{~h}$ at $37{ }^{\circ} \mathrm{C}$ and were subsequently washed three times using PBS. One hundred microliters of the secondary antibody, either HRP-conjugated anti-mouse IgG (diluted at $1: 10,000$ ) or HRP-conjugated anti-human IgG (diluted at 1:20,000), was added to each well. The wells were incubated for $1 \mathrm{~h}$ at $37{ }^{\circ} \mathrm{C}$ and were subsequently washed three times with PBS. The antigen-antibody reaction was detected by adding $100 \mu \mathrm{l}$ of HRP substrate, tetramethylbenzidine (TMB) (KPL Inc., Gaithersburg, MD, USA), to the wells, and the enzymatic reaction was allowed to proceed for $10 \mathrm{~min}$. The reaction was stopped by adding $50 \mu \mathrm{l}$ of $1 \mathrm{M}$ phosphoric acid, and 
the absorbance at $450 \mathrm{~nm}$ was measured using a microplate reader (Corona Electric, Ibaraki, Japan).

\section{Statistical analysis}

The sensitivity and specificity were analyzed using the MedCalc software program (version 16.4.3, MedCalc Software, Ostend, Belgium; https://www.medcalc.org; 2016).

\section{Results}

\section{Purification and antigenicity of rSjCatB}

The complete coding sequence of $\mathrm{SjCatB}$ was amplified by a PCR from cDNA synthesized from adult worm RNA and was cloned into the pCR plasmid vector for sequencing. The coding sequence was $1045 \mathrm{bp}$ in size (Fig. 1a, b). The identity with the GenBank deposited sequence (X70968.1) was confirmed (data not shown). The coding sequence was then subcloned into pET 28a (+) plasmid vector for the recombinant protein expression in $E$. coli with isopropyl-thio- $\beta$-Dgalactoside (IPTG) induction. The Ni-NTA column-purified $\mathrm{rSjCatB}$ was recognized as the strongest band seen in SDSPAGE with a molecular mass of $\sim 39 \mathrm{kDa}$, which was in good accordance with the size predicted from the $\mathrm{SjCatB}$ amino acid sequence (Fig. 1c).

\section{Serological evaluation of recombinant antigen}

The diagnostic potential of $\mathrm{rSjCatB}$ was evaluated in an ELISA with sera collected from experimentally infected mice. a

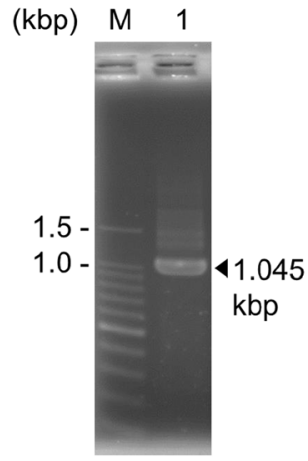

b

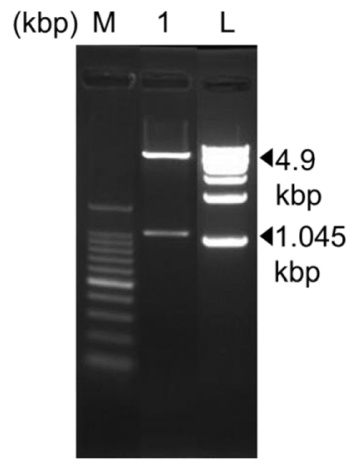

e

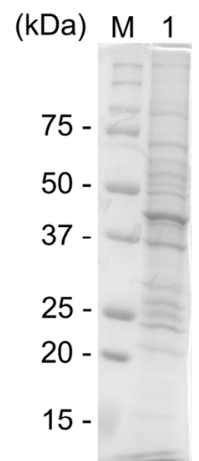

C

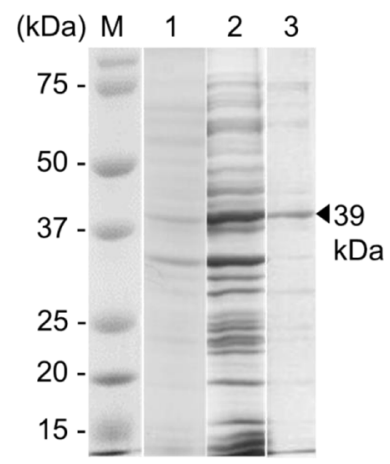

f

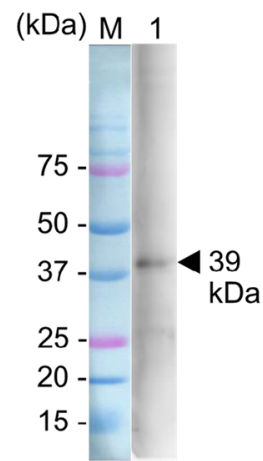

d

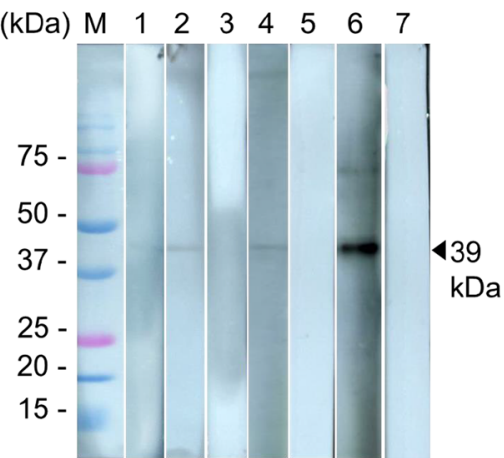

Fig. 1 Gel electrophoresis and SDS-PAGE of rSjCatB. a The PCRamplified DNA fragment coding for SjCatB. Lane 1, DNA for the SjCatB coding sequence amplified from the $S$. japonicum adult worm cDNA; M, marker. The triangle indicates the DNA fragment with a size of $1.045 \mathrm{kbp}$. b Recombinant plasmids with the SjCatB coding sequence. Lane 1, the recombinant plasmid with enzyme digestion. The upper band represents the plasmid DNA, and the lower band represents DNA for the SjCatB coding sequence. $\mathrm{M}$ and $\mathrm{L}, 100 \mathrm{bp}(\mathrm{M})$ and $\mathrm{kbp}(\mathrm{L})$ ladder markers. The triangles indicate DNAs with a size of $4.9 \mathrm{kbp}$ and $1.045 \mathrm{kbp}$, respectively. $\mathbf{c}$ The expression and purification of $\mathrm{rSjCatB}$. M, marker; lane 1, E. coli culture before adding IPTG; lane 2, E. coli culture after adding IPTG; lane $3, E$. coli culture after purification with

Ni-NTA column chromatography. The triangle indicates the polypeptide of $39 \mathrm{kDa}$ in size. $\mathbf{d}$ The recognition of $\mathrm{rSjCatB}$ on western blotting. M, marker; lane 1, anti-6xHis-tag mAb; lane 2, serum collected from an experimentally infected rabbit; lane 4 , serum collected from an experimentally infected mouse; lane 6, serum collected from a stool-positive patient; lanes 3, 5 and 7, rabbit, mouse, and human negative control sera. e SDS-PAGE of $S$. japonicum cercaria lysate. M, marker; lane 1, cercaria lysate stained with Coomassie Brilliant Blue R-250 (CBBR). f The recognition of $\mathrm{SjCatB}$ from the cercaria lysate using mouse antibody raised against $\mathrm{rSjCatB}$. M, marker; lane 1, a single band of $39 \mathrm{kDa}$ corresponding to $\mathrm{SjCatB}$ 
Twenty negative serum samples from normal uninfected mice were used to determine the cutoff value mean as the optical density (OD) value plus 2-fold standard deviation (SD), and the value was determined to be 0.075 . Using this cutoff value, all 20 experimentally infected mice were determined to be positive (sensitivity $=100 \%$ ), while 19 of the 20 normal uninfected mice were determined to be negative $($ specificity $=$ 95.0\%) (Fig. 2a).

The sera collected from experimentally infected mice at 0 , $1,2,3,4,5,6$, and 7 weeks post-infection were evaluated for the antibody response against $\mathrm{rSjCatB}$ in ELISA. From as early as 6 weeks post-infection, sera from 2 of the 3 experimentally infected mice showed OD values higher than the cutoff value of 0.091 . Sera from normal uninfected mice did not show OD values higher than the cutoff value (Fig. $2 b$ ).

The diagnostic potential of $\mathrm{rSjCatB}$ in an ELISA with sera from human clinical cases was also evaluated. Thirty serum samples from normal healthy US volunteers were used to determine the cutoff value $(\mathrm{OD}+2 \mathrm{SD})$, which was calculated to be 0.33 . Using this cutoff value, 26 of 30 stool-confirmed
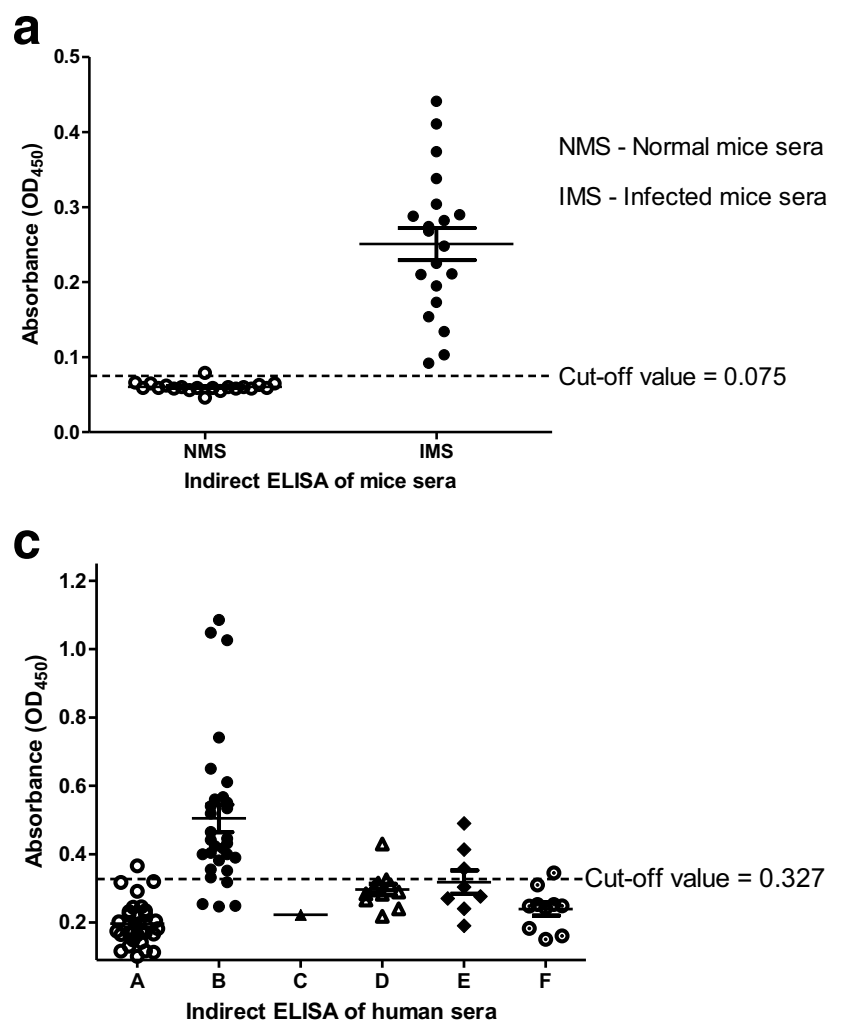

Fig. 2 The ELISA results with $\mathrm{rSjCatB}$. a The results with mouse sera. NMS, serum samples collected from normal uninfected mice $(n=20)$; IMS, serum samples collected from mice experimentally infected with the parasite $(n=20)$. The dotted line across the graph indicates the cutoff value $=0.075$. The thin solid line in each group shows the mean absorbance, while the thick solid lines represent the SD. b The results of the evaluation of antibody response against SjCatB. Each symbol shows the mean absorbance of the triplicate readings for the serum samples collected from normal $(n=2)$ and parasite-infected mice $(n=3)$ clinical cases were determined to be positive (sensitivity $=$ $86.7 \%$ ), while 29 of 30 US volunteers were determined to be negative (specificity $=96.7 \%$ ) (Fig. 2c).

A T. trichiura-positive sample showed no cross-reaction with the recombinant antigen. In contrast, 1 of the 11 $P$. westermani-positive samples, 3 of the $8 O$. viverrini-positive samples, and 1 of the $10 \mathrm{E}$. histolytica-positive samples showed OD values that were slightly higher than the cutoff value (Fig. 2c). Statistical analysis revealed that there is a significant difference with the antibodies detected from uninfected individuals as compared with those infected with other parasites (data not shown).

Furthermore, rSjCatB was recognized in western blotting as a $39 \mathrm{kDa}$ polypeptide with anti-6xHis-tag mAb as well as serum samples collected from experimentally infected animals and the stool-positive patients but not with normal serum samples (Fig. 1d). The antibodies raised against $\mathrm{rSjCatB}$ were able to recognize the enzyme as a $39 \mathrm{kDa}$ polypeptide with western blotting in $S$. japonicum cercaria lysate (Fig.1f) which had been

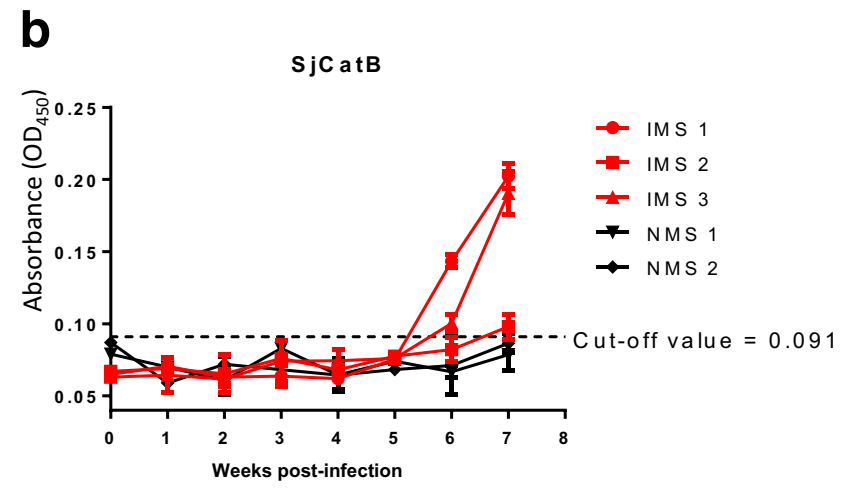

at $0,1,2,3,4,5,6$, and 7 weeks post-infection. The dotted line across the graph indicates the cutoff value $=0.091$. $\mathrm{c}$ The results with human sera. A, negative serum samples from US volunteers $(n=30)$; B, serum samples from $S$. japonicum stool-positive patients $(n=30)$; C, serum-positive for T. trichiura $(n=1)$; D, serum-positive for $P$. westermani $(n=11)$; E, serum-positive for $O$. viverrini $(n=8)$; F, serum-positive for $E$. histolytica $(n=10)$. The dotted line across the graph indicates the cutoff value $=$ 0.327 . The thin solid line in each group shows the mean absorbance 
separated with SDS-PAGE (Fig. 1e). The results suggested that the recombinant protein was antigenically similar to the native protein.

\section{Discussion}

The current, most used diagnostic test for S. japonicum infection is stool microscopy, which is highly specific and easy to perform (Katz et al. 1972). However, the test has a disadvantage in detecting early infection and infection with low parasite burden (Pontes et al. 2003; $\mathrm{Yu}$ et al. 2007; Zhu 2005). In order to increase the sensitivity of the test, several modifications including concentration of eggs in the stool using the formalinether concentration technique (FECT) and collection of stool samples over several consecutive days have been applied (Utzinger et al. 2001; Booth et al. 2003; Ridley and Hawgood 1956). However, these modifications are too laborious in large-scale nationwide surveys (Weerakoon et al. 2015). Although point-of-care diagnostic test (POCT) kits are available for other schistosome species, sensitivities of these kits in the diagnosis of $S$. japonicum infection were unsatisfactory (van Dam et al. 2015). Moreover, the diagnostic performance of POC-CCA which is a POCT to detect $S$. mansoni infection correlates with the intensity of infection and its sensitivity may decrease in cases with a low parasite burden, similar to stool microscopy (Stothard et al. 2006; Tchuem Tchuenté et al. 2012; Legesse and Erko 2007). An ELISA with the recombinant antigen that can detect early infection with high sensitivity and specificity may provide an alternative mean which can be a reliable replacement of stool microscopy and POCT for detecting $S$. japonicum infection in large-scale nationwide surveys.

Cathepsin B (CatB) is a lysosomal cysteine protease found in the gut of schistosome parasite. The parasite uses $\mathrm{CatB}$ as a major enzyme for nutrient acquisition through hemoglobin metabolism (Caffrey et al. 2004). CatB is identified in the acetabular gland as the main enzyme in the cercarial stage of $S$. japonicum. The parasite uses the enzyme to dissolve collagen IV, fibronectin, and laminin in the extracellular matrix of the host skin during penetration (Dvorak et al. 2008; Curwen and Wilson 2003). The abundant expression of the enzyme in the cercarial stage of $S$. mansoni particularly in the cecum and protonephridia is also reported (El Ridi et al. 2014; Skelly and Shoemaker 2001). The crucial role of this enzyme in the parasite makes it an ideal target for drug and vaccine development (Smooker et al. 2010; Wasilewski et al. 1996). The early exposure of CatB to the host's immune system also implies an early immune response against this enzyme. Hence, this antigen may have an advantage as a potential marker for the early antibody-based diagnosis of the infection.

In the present study, an ELISA using rSjCatB showed high sensitivity and specificity in detecting the parasite infections both in experimentally infected mice and in stool-confirmed patients. The ELISA data also suggested a potential application of this antigen in the early diagnosis of the infection. Compared with SjTPx-1 which detected only 1 of 3 experimentally infected mice at 6 weeks post-infection (Fig. S1), rSjCatB detected 2 of 3 experimentally infected mice at 6 weeks post-infection. Here, we observed a varying antibody response with $\mathrm{rSjCatB}$ that can be attributed to the fact that there is a possibility of different infectivity levels of these mice post cercarial challenge (Maloney and Webbe 1982). Furthermore, antibodies against $\mathrm{rSjCatB}$ were detected at 6 weeks post-infection before the parasite fully develops into an adult and starts to lay eggs. This finding validates the data from previous studies with $S$. mekongi CatB (Sangfuang et al. 2016) and S. mansoni CatB (Ruppel et al. 1985a, b), in which specific antibodies were detected from as early as 4 weeks post-infection. Early detection is beneficial in cases of acute infection, such as in tourist potentially exposed to the disease where eggs of the parasite are not detected in the stool beyond 8 weeks post-infection. Earlier diagnosis could provide travelers suspected of exposure with early access to treatment and thus prevent the introduction of the disease into an uncontaminated environment as well as the pathological damage induced by egg deposition in vital organs. The results of the present study showed that SjCatB has high sensitivity and specificity in the diagnosis of $S$. japonicum infection, even in the early stages of infection, with minimal cross-reactivity with other parasitic infections.

The environmental changes brought about by the development of water resources, such as the building of dams, which introduces the intermediate snail hosts, as well as the migration of patients and reservoir animals into other previously non-endemic areas, may give rise to new endemic foci (Patz et al. 2000; Ross et al. 2001). Effective screening of individuals and animals moving from schistosomiasis-endemic areas may prevent the introduction of the disease in non-endemic areas. Thus, a sensitive and specific test that can detect earlystage schistosomiasis infection may have an advantage over the traditional diagnostic methods that are currently applied and may in turn improve elimination programs that are currently set in place.

Taken together, $\mathrm{SjCatB}$, which showed high sensitivity and specificity in the detection of $S$. japonicum infection may be a promising serodiagnostic antigen. To facilitate its field application, additional experiments with larger numbers of samples from humans and animals are currently underway. 
Acknowledgments We thank Dr. Hiroshi Ohmae of the National Institute of Infectious Diseases, Japan, for providing the schistosomiasis-positive serum samples; Dr. Isao Nagano of the Department of Parasitology, Gifu University Graduate School of Medicine for the O. viverrini-positive samples; Dr. Haruhiko Maruyama of Miyazaki University Graduate School of Medicine for the P. westermani-positive samples.

\section{Compliance with ethical standards}

Conflict of interest The authors declare that they have no conflict of interest.

Ethical approval All procedures performed in studies involving animals were in accordance with the ethical standards of Obihiro University of Agriculture and Veterinary Medicine, Japan, animal committee (approval no. 29-52).

\section{References}

Angeles JMM, Goto Y, Kirinoki M, Asada M, Leonardo LR, Rivera PT, Villacorte EA, Inoue N, Chigusa Y, Kawazu S (2012) Utilization of ELISA using thioredoxin peroxidase-1 and tandem repeat proteins for diagnosis of Schistosoma japonicum infection among water buffaloes. PLOS Negl Trop Dis 6:e1800

Booth M, Vounatsou P, Ngoran EK, Tanner M, Utzinger J (2003) The influence of sampling effort and the performance of the Kato-Katz technique in diagnosing Schistosoma mansoni and hookworm coinfections in rural Côte d'Ivoire. Parasitology 127:525-531

Caffrey CR, McKerrow JH, Salter JP, Sajid M (2004) Blood 'n'guts: an update on schistosome digestive peptidases. Trends Parasitol 20: 241-248

Centers for Disease Control website https://www.cdc.gov/parasites/ schistosomiasis/health professionals/index.html Accessed 25 August, 2017

Chitsulo L, Engels D, Montresor A, Savioli L (2000) The global status of schistosomiasis and its control. Acta Trop 77:41-51

Curwen RS, Wilson RA (2003) Invasion of skin by schistosome cercariae: some neglected facts. Trends Parasitol 19(2):63-66 discussion 66-8

Dvorak J, Mashiyama ST, Braschi S, Sajid M, Knudsen GM, Hansell E, Lim KC, Hsieh I, Bahgat M, Mackenzie B, Medzihradszky KF, Babbitt PC, Caffrey CR, McKerrow JH (2008) Differential use of protease families for invasion by schistosome cercariae. Biochimie 90(2):345-358

El Ridi R, Tallima H, Dalton JP, Donnelly S (2014) Induction of protective immune responses against schistosomiasis using functionally active cysteine peptidases. Front Genet 5:119

González AY, Sulbarán GS, Ballen DE, Cesari IM (2015) Immunocapture of circulating Schistosoma mansoni cathepsin B antigen (Sm31) by anti-Sm31 polyclonal antibodies. Parasitol Int 65(3):191-195

Karcher H, Loddenkemper C, Zeitz M, Schneider T (2008) Intestinal schistosomiasis in a traveler returning from Rwanda and Burundi. Int J Color Dis 23:1021-1022

Katz N, Chaves A, Pellegrino J (1972) A simple device for quantitative stool thick-smear technique in schistosomiasis mansoni. Rev Inst Med Trop Sao Paulo 14:397-400

Legesse M, Erko B (2007) Field-based evaluation of a reagent strip test for diagnosis of Schistosoma mansoni by detecting circulating cathodic antigen in urine before and after chemotherapy. Trans R Soc Trop Med Hyg 101:668-673

Leonardo L, Rivera P, Saniel O, Antonio Solon J, Chigusa Y, Villacorte E, Christopher Chua J, Moendeg K, Manalo D, Chrisostomo B, Sunico L, Boldero N, Payne L, Hernandez L, Velayudhan R (2015) New endemic foci of schistosomiasis infections in the Philippines. Acta Trop 141:354-360

Liu S, Cai P, Piao X, Hou N, Zhou X, Wu C, Wang H, Chen Q (2014) Expression profile of the Schistosoma japonicum degradome reveals differential protease expression patterns and potential antischistosomal intervention targets. PLoS Comput Biol 10(10): e1003856

Mahmoud AAF (2001) Schistosomiasis. Tropical Medicine Science and Practice, vol 3. Singapore, Imperial College Press

Maloney NA, Webbe G (1982) A rapid method for the infection of laboratory mice with Schistosoma japonicum. Trans R Soc Trop Med Hyg 76(2):200-203

Patz JA, Graczyk TK, Geller N, Vittor AY (2000) Effects of environmental change on emerging parasitic diseases. Int J Parasitol 30:13951405

Pontes LA, Oliveira MC, Katz N, Dias-Neto E, Rabello A (2003) Comparison of a polymerase chain reaction and the Kato-Katz technique for diagnosing infection with Schistosoma mansoni. Am J Trop Med Hyg 68:652-656

Ridley DS, Hawgood BC (1956) The value of formol-ether concentration of faecal cysts and ova. J Clin Pathol 9:74-76

Ross AG, Sleigh AC, Li Y, Davis GM, Williams GM, Jiang Z, Feng Z, McManus DP (2001) Schistosomiasis in the People's Republic of China: prospects and challenges for the 21 st century. Clin Microbiol Rev 14:270-295

Ruppel A, Diesfeld HJ, Rother U (1985a) Immunoblot analysis of Schistosoma mansoni antigens with sera of schistosomiasis patients: diagnostic potential of an adult schistosome polypeptide. Clin Exp Immunol 62:499-506

Ruppel A, Rother U, Vongerichten H, Lucius R, Diesfeld HJ (1985b) Schistosoma mansoni: immunoblot analysis of adult worm proteins. Exp Parasitol 60:195-206

Ruppel A, Shi YE, Wei DX, Diesfeld HJ (1987) Sera of Schistosoma japonicum-infected patients cross-react with diagnostic $31 / 32 \mathrm{kD}$ proteins of S. mansoni. Clin Exp Immunol 69:291-298

Sangfuang M, Chusongsang Y, Limpanont Y, Vanichviriyakit R, Chotwiwatthanakun C, Sobhon P, Preyavichyapugdee N (2016) Schistosoma mekongi cathepsin B and its use in the development of an immunodiagnosis. Acta Trop 155:11-19

Skelly PJ, Shoemaker CB (2001) Schistosoma mansoni proteases Sm31 (cathepsin B) and Sm32 (legumain) are expressed in the cecum and protonephridia of cercariae. J Parasitol 87:1218-1221

Smooker PM, Jayaraj R, Pike RN, Spithill TW (2010) Cathepsin B proteases of flukes: the key to facilitating parasite control? Trends Parasitol 26:506-514

Stothard JR, Kabatereine NB, Tukahebwa EM, Kazibwe F, Rollinson D, Mathieson W, Webster JP, Fenwick A (2006) Use of circulating cathodic antigen (CCA) dipsticks for detection of intestinal and urinary schistosomiasis. Acta Trop 97:219-228

Tchuem Tchuenté LA, Kueté Fouodo CJ, Kamwa Ngassam RI, Sumo L, Dongmo Noumedem C, Kenfack CM, Gipwe NF, Nana ED, Stothard JR, Rollinson D (2012) Evaluation of circulating cathodic antigen (CCA) urine-tests for diagnosis of Schistosoma mansoni infection in Cameroon. PLoS Negl Trop Dis 6:e1758

Utzinger J, Booth M, N'Goran EK, Muller I, Tanner M, Lengeler C (2001) Relative contribution of day-to-day and intra-specimen variation in faecal egg counts of Schistosoma mansoni before and after treatment with praziquantel. Parasitol 122:537-544

van Dam GJ, Odermatt P, Acosta L, Bergquist R, de Dood CJ, Kornelis D, Muth S, Utzinger J, Corstjens PL (2015) Evaluation of banked urine samples for the detection of circulating anodic and cathodic antigens in Schistosoma mekongi and S. japonicum infections: a proof-of-concept study. Acta Trop 141:198-203

Wasilewski MM, Lim KC, Phillips J, McKerrow JH (1996) Cysteine protease inhibitors block schistosome hemoglobin degradation 
in vitro and decrease worm burden and egg production in vivo. Mol Biochem Parasitol 81:179-189

Weerakoon KG, Gobert GN, Cai P, McManus DP (2015) Advances in the diagnosis of human schistosomiasis. Clin Microbiol Rev 28:939967

Yu JM, de Vlas SJ, Jiang QW, Gryseels B (2007) Comparison of the Kato-Katz technique, hatching test and indirect hemagglutination assay (IHA) for the diagnosis of Schistosoma japonicum infection in China. Parasitol Int 56:45-49

Zhang Y, Zhao J, Wang X, Xu X, Pan W (2015) Evaluation of six novel antigens as potential biomarkers for the early immunodiagnosis of schistosomiasis. Parasit Vectors 8:447. https://doi.org/10.1186/ s13071-015-1048-2

Zhou XH, Wu JY, Huang XQ, Kunnon SP, Zhu XQ, Chen XG (2010) Identification and characterization of Schistosoma japonicum Sjp40, a potential antigen candidate for the early diagnosis of schistosomiasis. Diagn Microbiol Infect Dis 67:337-345

Zhu YC (2005) Immunodiagnosis and its role in schistosomiasis control in China: a review. Acta Trop 96:130-136

Publisher's note Springer Nature remains neutral with regard to jurisdictional claims in published maps and institutional affiliations. 